\title{
The management of head and neck cancer in Africa. What lessons can be learned from African literature?
}

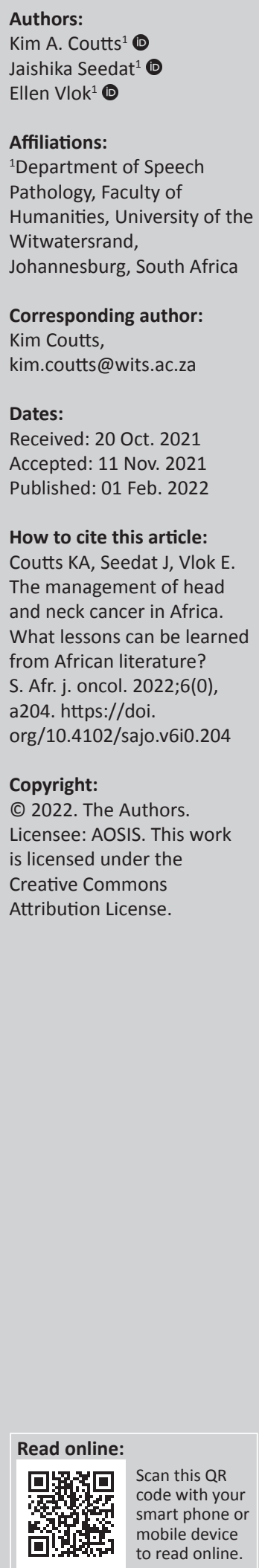

There is a significant dearth of contextually relevant information related to the management of head and neck cancer (HNC) in Africa. The aim of this letter was to put forward the findings from our larger systematic review to describe the current management of HNC patients in Africa and to identify gaps and present potential solutions. Sixty-six articles were included and analysed with descriptive statistics, a narrative synthesis, and thematic analysis. Surgical resection remains the primary medical intervention in Africa, whilst chemotherapy and radiation services remain limited. There was no mention of multidisciplinary team input in the management of these patients, including no description of any rehabilitative treatments. There are significant resource shortages ranging from access to medical equipment to both skilled medical and rehabilitative staff. The findings from this study imply that the management of $\mathrm{HNC}$ in Africa requires a possible transdisciplinary approach to improve access to services. Health professionals also need to explore a community-based level approach to care to improve access. There needs to be more context-specific research to improve contextually relevant teaching and practice in HNC.

Keywords: head and neck cancer; rehabilitation; management; speech language pathology; interdisciplinary; dysphagia.

\section{Introduction}

Cancer is the second leading cause of death globally ${ }^{1}$ with low to middle income countries (LMICs) accounting for $70 \%$ of those deaths. ${ }^{2}$ However, there appears to be a significant dearth of information related to cancer studies in Africa. ${ }^{2}$ This scientific letter will highlight how the lack of data can impact on the treatment methodologies and protocols used in Africa specifically for head and neck cancer (HNC) and identify ways forward to make them contextually appropriate.

The majority of the data around HNC stems from economically developed countries, which present with significantly different patient demographics and resource availability, which has implications for the medical, surgical and rehabilitative treatment of HNC in the African context.

This letter stems from a larger systematic review that used a mixed methods explanatory design to review 66 articles. The data was analysed both quantitatively and qualitatively. The quantitative data was further explained by means of thematic analyses. The review included articles relating to $\mathrm{HNC}$ and then analysed the results according to the sub-categories such as oral cancer, oropharyngeal cancer and nasopharyngeal caner. The results are discussed in relation to the themes that emerged.

\section{Surgical treatment}

Surgery was mentioned as the primary means for oropharyngeal cancer specifically, and continues to remain the primary treatment for HNC in Africa with $44 \%$ of the patients in the review articles undergoing surgery. The articles did not mention if the intention of the surgery was for curative or palliative purposes. Thematically, the primary reason stated for surgical intervention in Africa was because of the lack of radiation or chemotherapy services within certain regions.

\section{Challenges with radiation and chemotherapy}

Only four countries (Tanzania, South Africa, Uganda and Nigeria) reported having access to radiotherapy. This concluded that waiting times to receive radiation were excessively long. Another factor reported from Nigeria, was the lack of affordability of these services because of lack of health insurance. Thematically, poor access to hospitals was a significant challenge, as patients often lived far away from the treating facilities that had the appropriate resources for management. All of these factors are also pertinent in South Africa. 
Chemotherapy services were documented predominantly for HNC patients. Thematically, chemotherapy was also a challenge as a result of lack of access and poor patient compliance. Reasons for these challenges included: a lack of medical oncologists proving chemotherapy; poor financial affordability of the chemotherapy medication; and patients often resorting to traditional medicine. ${ }^{3,4,5}$ In terms of radiation, there is limited staff availability to use this equipment, as well as inadequate training and skills related to administering dosages. ${ }^{6}$

\section{Traditional medicine}

In the articles, $15 \%(n=220)$ of the participants who commenced treatment sought out a traditional healer first. In Uganda, traditional medicine was sought out first ${ }^{6}$ by $84.3 \%$ of the participants as 'Traditional herbalists in western Uganda are affordable and easy to access, and they understand cultural beliefs'. Being a continent of diverse ethnicities and cultures, further exploration of how traditional health seeking management versus western practices pertaining to $\mathrm{HNC}$ in Africa is needed; and this may explain aspects such as late diagnosis. Insights into the knowledge of the traditional versus western medicine would assist in understanding underlying reasons around the management of HNC in Africa specifically.

\section{Multidisciplinary team management}

None of the articles identified multidisciplinary team management (MDT) members involved in HNC treatment in Africa. This includes the rehabilitation of these patients postsurgery. This is concerning. Limited access to the appropriate treatment modalities at an early phase will have a significant impact later on as when patients present later, they have more complications. This has implications for both medical, surgical and rehabilitative staff. The MDT need to account for these further complications in both the acute and chronic phases of HNC. This is a topic that needs to be further explored for the African context. Data on practice patterns relating to the long-term rehabilitation of HNC patients in Africa are also clearly warranted.

A strong theme that emerged was the lack of healthcare infrastructure across Africa. This needs to be considered by professionals and a consideration to possibly shift towards a community-based therapy over hospital-based therapy ${ }^{7}$ approach when it comes to rehabilitation services as well as post-surgical follow ups. This could improve access to the patients, and follow up services, prior to, during, and after treatment.

Given the limited access to professionals and cancer services in Africa, perhaps professionals working in HNC should adopt a transdisciplinary approach. In this model, the team working with the patient has a common goal, as well as a common treatment plan, regardless of the discipline. ${ }^{8}$ Essentially, each discipline shares each other's roles and responsibilities thus making treatment more time and cost-effective. This approach can offset the few number of professionals who are available to assist in the treatment of HNC and can also aid in improving general access to services. ${ }^{9}$ While this approach may be required, it may present with challenges such as the lack of professionals providing the initial training and surface level treatment.

\section{Conclusion}

There are multiple challenges facing people with HNC in Africa including, poor access to the appropriate healthcare and insufficient, and/or skilled staff to care for these patients. There is also limited contextually relevant data on HNC in Africa which limits the ability to build context-specific guidelines and policies. This letter has been able to identify important research gaps when it comes to the management of HNC in Africa that will assist in driving a way forward for teaching, research and practice.

\section{Acknowledgements Competing interests}

The authors declare that no competing interest exists.

\section{Authors' contributions}

K.C. wrote the full article and J.S. contributed toward the editing and writing of the article. This work is based on the Masters thesis of E.V.

\section{Ethical considerations}

Ethical clearance was obtained from the University of the Witwatersrand Human Research Ethics Committee (Wits HREC M1911136), on the 09 December 2019.

\section{Funding information}

This article will be funded by the Faculty of Humanities, University of the Witwatersrand.

\section{Data availability}

Data are available on request from the corresponding author, C.A.T.

\section{Disclaimer}

The views and opinions expressed in this article are those of the authors and do not necessarily reflect the official policy or position of any affiliated agency of the authors.

\section{References}

1. Plummer M, De Martel C, Vignat J, Ferlay J, Bray F, Franceschi S. Global burden of cancers attributable to infections in 2012: A synthetic analysis. Lancet Glob Heal. 2016;4(9):e609-e616. https://doi.org/10.1016/S2214-109X(16)30143-7

2. Ngoma T, Ngoma M. Cancer control in Africa: Is cancer research a luxury or necessity? Ecancermedicalscience. 2019;13:947. https://doi.org/10.3332/ ecancer.2019.947

3. Dos Passos G, Rogers $A D$, Price $C E$, et al. Loupe magnification for head and neck free flap reconstruction in a developing country. Eur J Plast Surg. 2015;38(5):363-370. https://doi.org/10.1007/s00238-015-1108-z 
4. Gilyoma JM, Rambau PF, Masalu N, Kayange NM, Chalya PL. Head and neck cancers: A clinico-pathological profile and management challenges in a resource-limited A clinico-pathological profile and management challenges in a resource-limited
setting. BMC Res Notes. 2015;8(1):1-9. https://doi.org/10.1186/s13104-015-1773-9

5. Kakande E. Head and neck squamous cell carcinoma in Western Uganda: Disease of uncertainty and poor prognosis. OTO Open. 2018:2(1):219-225. https://doi.org/ $10.1177 / 2473974 X 18761868$

6. Rosenblatt E, Zubizarreta E, editors. Radiotherapy in cancer care: Facing the global challenge. International Atomic Energy Agency (IAEA) [hompage on the Internet] 2017 [cited 2021 Aug 25]. Available from: https://www.iaea.org/publications/ 10627/radiotherapy-in-cancer-care-facing-the-global-challenge
7. King $G$, Strachan $D$. The application of a transdisciplinary model for early intervention services. Infants Young Child, 22(3):211-223. https://doi.org/10.1097/IYC 0b013e3181abe1c3

8. Faggan, J. Workforce considerations, training, and diseases in Africa. Otolaryngol Clin North Am. 2018;51(3):643-649. https://doi.org/10.1016/j. otc.2018.01.009

9. Beaudoim P. Barriers in access to care for patients with head and neck cancer in resource-limited settings: A systematic review. JAMA Otolaryngo Head Neck Surg. 2020;146(3):291-297. https://doi.org/10.1001/jamaoto. 2019.4311 